\title{
The implement of a remote control system for automated warehouse by ZigBee networking
}

\author{
Ho-Chih Cheng $^{1, *}$ and Min-Chie Chiu ${ }^{1}$ \\ ${ }^{1}$ Department of Mechanical and Automation Engineering, Chung Chou University of Science and Technology, 6, Lane 2, Sec.3, \\ Shanchiao Rd., Yuanlin, Changhua 51003, Taiwan, R.O.C.
}

\begin{abstract}
Excessive raw materials and products in stock unduly increase an enterprise's overhead. However, insufficient raw materials and products will also influence the efficiency and output of the manufacturing process. Therefore, to largely reduce stock cost and facilitate adequate production for plants with multiple manufacturing processes and warehouses, the adjustment of raw material and products between warehouses is necessary. In addition, in order to keep track of current raw materials and products, remote online monitoring and control of the storage/retrieval system is compulsory. To simplify the search in an AS/BS process, a graph-aided strategy that manually selects the location within the stock map is presented. Moreover, to avoid interference from internet wiring allocation with existing equipment, a wireless storage/retrieval system using the ZigBee module is adopted. Consequently, a prototype of the remote wireless monitoring and control storage/retrieval system for sixteen storage spaces is exemplified.
\end{abstract}

\section{Introduction}

It is important to have an automated warehouse in the modern manufacturing field $[1,2]$. From a mechanical perspective, a 3-D automated warehouse will save space; therefore, an assortment of automated warehouses has been developed $[3,4]$. The automated Storage/Retrieval System (AS/RS) used in manufacturing, warehousing, and distribution applications is an efficient system. Order selection is fundamental for the retrieval function in automated warehouses. However, it is costly and timeconsuming [5]. Many researchers have assessed the order selection problems in an Automated Storage/Retrieval System (AS/RS) and in automated warehousing systems. Ratliff and Rosenthal [6] proposed a graph-based algorithm to find the shortest path for selecting location. Linn and Wysk [7] proposed an expert-system-based AS/RS controller to search for storage and retrieval. Egbelu [8] minimized the service response time in the AS/RS by selecting the dwell point of the storage/retrieval machine. Mansuri [9] proposed a computerized algorithm to find the dedicated storage allocation.

However, for a plant with multiple manufacturing processes and warehouses, research for a more flexible and efficient distributing, monitoring, and controlling network has rarely been addressed. So, to reduce stock cost and to improve the manufacture process, a remotely distributing, monitoring, and controlling network to analyze online the status of the AS/RS and remotely adjust the stock is proposed. In addition, to simplify the search for an AS/BS process, a graph-aided method that manually selects the location within the stock map is presented. Moreover, to avoid interference from internet wiring allocation with existing equipment, a wireless storage/retrieval system using the ZigBee module is adopted. A prototype of the remote wireless monitoring and control storage/retrieval system with sixteen storage spaces (used for storage/retrieval of one kind of raw material) will be exemplified in this study. The related architecture for a distributing, monitoring, and controlling network for multiple AS/RS systems is shown in Fig. 1.

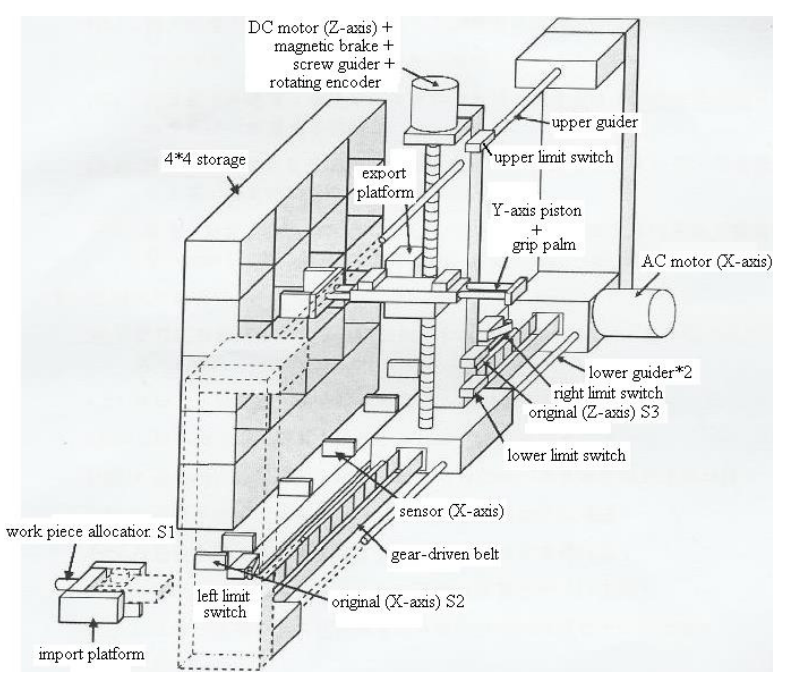

Fig. 1. The structure of the AS/RS system.

\section{Graph-aided location selection for the storage/retrieval process}

* Corresponding author: hccheng@gm.ccut.edu.tw 
The structure of the AS/RS system is depicted in Figs. 1 and 2. In addition, the hardware for the remotely distributing, monitoring, and controlling network for multiple AS/RS systems with sixteen storage spaces (used for storage/retrieval for one kind of raw material) is shown in Fig. 3. The structure of the server pc for an individual AS/RS system is depicted in Fig. 4.

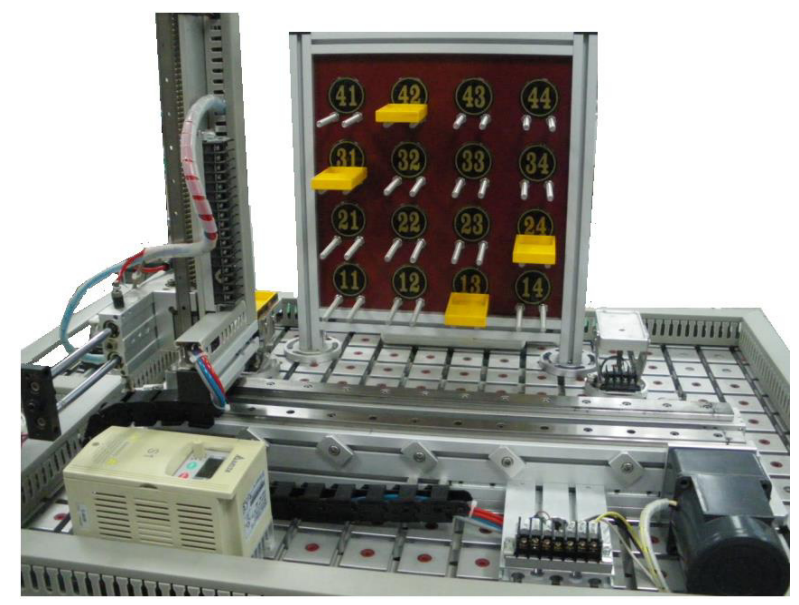

Fig. 2. The prototype of the remote distributing, monitoring, and controlling network for multiple AS/RS systems with sixteen storage spaces (used for storage/retrieval for one kind of raw material).

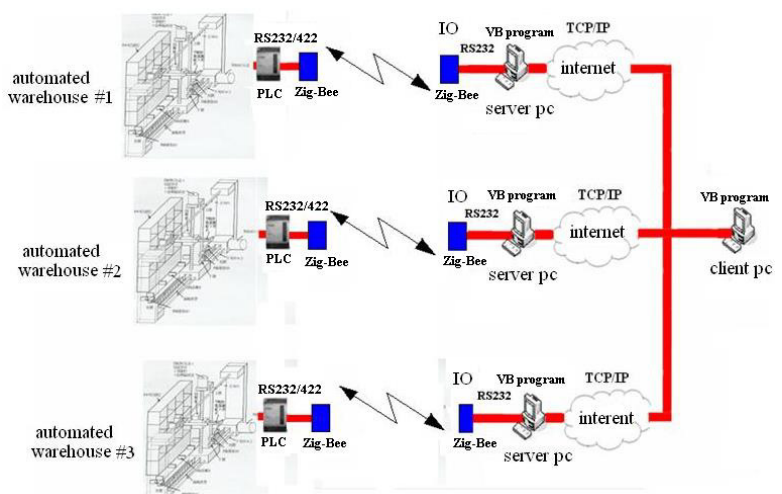

Fig. 3. The related architecture of the remote distributing, monitoring, and controlling network for multiple AS/RS systems.

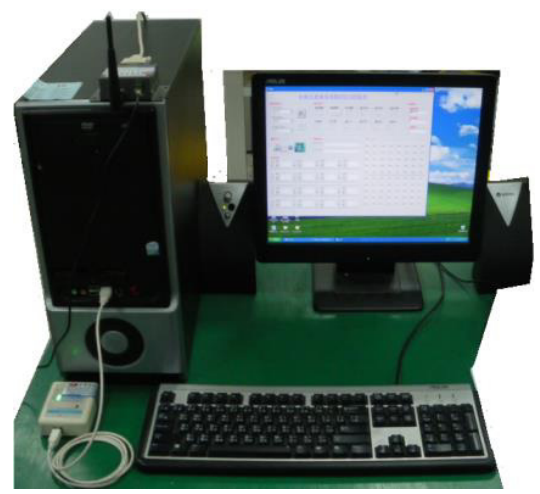

Fig. 4. The structure of the server pc for an individual AS/RS system.
The related wiring diagram of the PLC connection is shown in Fig. 5. In order to remotely manipulate the AS/BS system via the server pc, the auxiliary relays defined in Table 1 have been added into the PLC program. As indicated in Fig. 6, the auxiliary relays remarked in red are in place of the button functions. The server pc can then submit the command to actuate the related function of the AS/BS system.

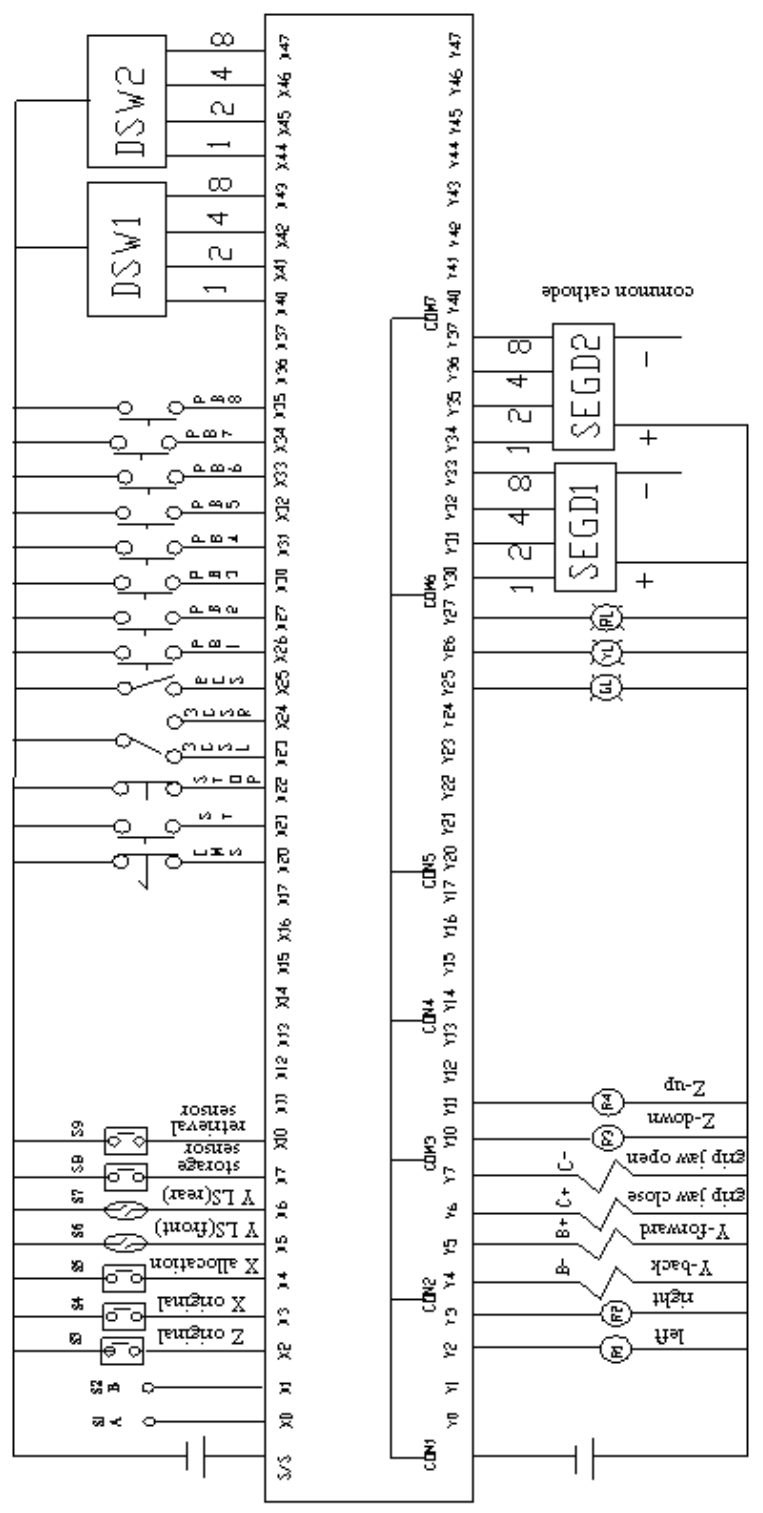

Fig. 5. The related wiring diagram of the PLC connection. 


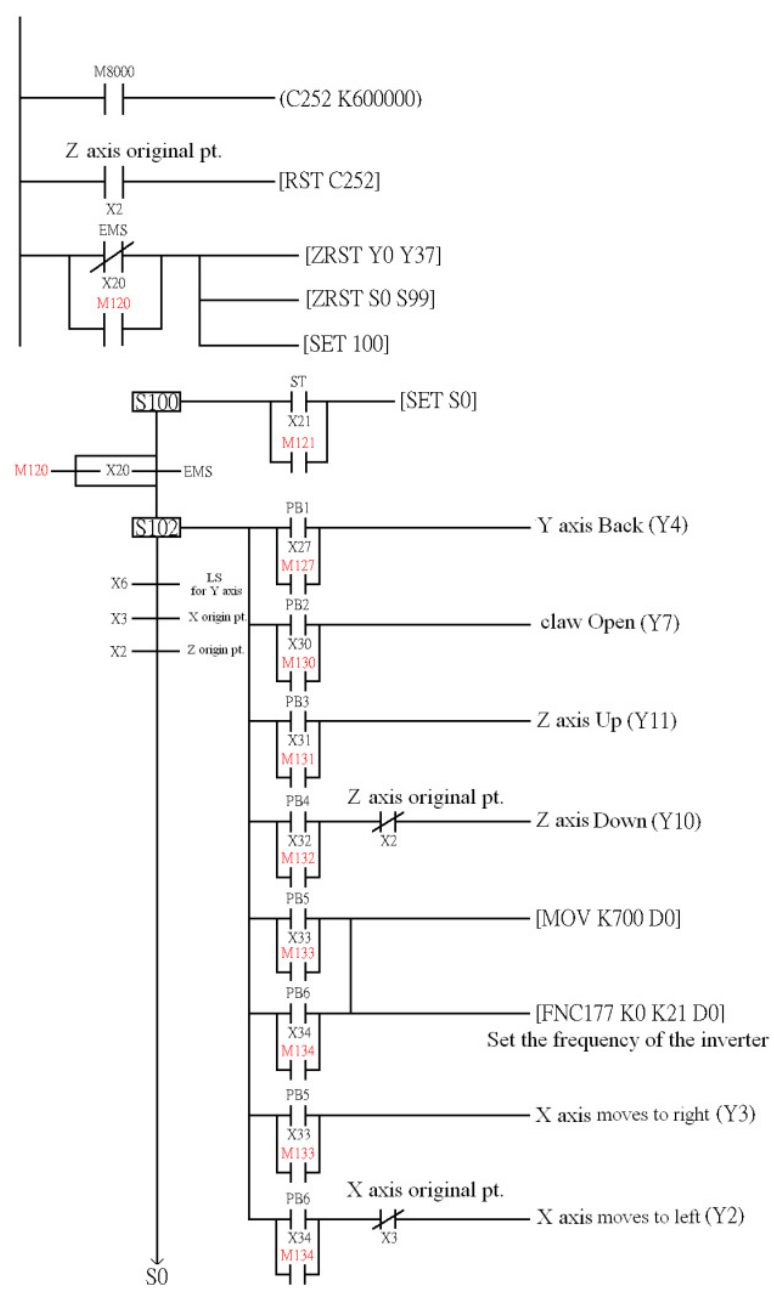

Fig. 6. An abstract of the ladder program built in the PLC used to actuate the AS/RS system by the auxiliary relays.

Table 1. The auxiliary relays used to remotely control the action of the PLC program.

\begin{tabular}{|c|c|c|}
\hline $\begin{array}{c}\text { Auxiliary Relay } \\
\text { Number }\end{array}$ & Action & PLC Program \\
\hline M120 & $\begin{array}{c}\text { Emergent } \\
\text { open/close }\end{array}$ & $\begin{array}{c}\text { SET }, \text { RST } \\
\text { M120 }\end{array}$ \\
\hline M121 & $\begin{array}{c}\text { Start the AS/BS } \\
\text { system }\end{array}$ & $\begin{array}{c}\text { SET }, \text { RST } \\
\text { M121 }\end{array}$ \\
\hline M123 & Object retrieval & $\begin{array}{c}\text { SET, RST } \\
\text { M123 }\end{array}$ \\
\hline M124 & Object storage & $\begin{array}{c}\text { SET }, \text { RST } \\
\text { M124 }\end{array}$ \\
\hline M127 & Y axis Back & $\begin{array}{c}\text { SET }, \text { RST } \\
\text { M127 }\end{array}$ \\
\hline M130 & Claw Open & $\begin{array}{c}\text { SET }, \text { RST } \\
\text { M130 }\end{array}$ \\
\hline M131 & Z axis Up & $\begin{array}{c}\text { SET, RST } \\
\text { M131 }\end{array}$ \\
\hline M132 & Z axis Down & SET, RST \\
& M132 \\
\hline M133 & X axis move to & SET, RST \\
& right & M133 \\
\hline M134 & X axis move to & SET RST \\
& left & \\
\hline
\end{tabular}

Moreover, the interface of the server pc programmed in VB is also illustrated in Fig. 7. In order to simplify the search for the AS/RS process, a graph-aided method that manually selects the location within the stock map is presented. As indicated in Fig. 7, there is a $4 \times 4$ stock map. Each location reveals the current status of the stock in the AS/RS system. A location with a red light $\mathrm{ON}$ represents an occupied storage space; therefore, only the retrieval operation can be executed. With the red light OFF, the storage location is empty and will be available for storage. Each location has coordinates and is built into the system program.

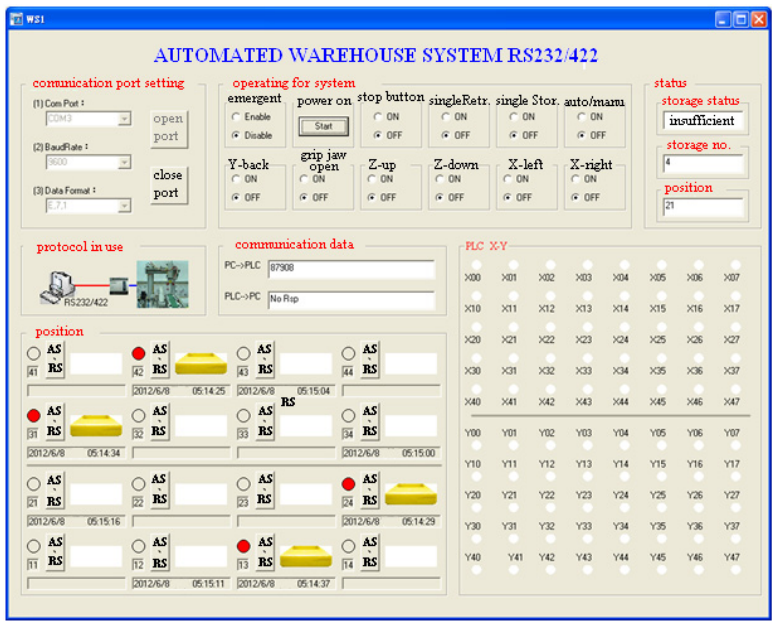

Fig. 7. The interface of the server pc programmed in VB.

\section{System established and communication format}

As indicated in Figs. 1 and 2, a PLC is used as a controller to actuate the actuators (an $\mathrm{X}$-axis, $\mathrm{Y}$-axis, and $\mathrm{Z}$-axis air-driven piston) of the storage/ retrieval mechanism. In addition, a grip palm is used to catch the object. There are two sensors used to detect the object at both the feeding and the output platforms. In order to monitor online the storage status and remotely control the storage/retrieval with a server $\mathrm{pc}$ at the control room of the individual AS/RS system, a wireless communication between the server pc and the AS/RS system is established using the ZigBee module connected to the PLC via RS232/RS422 protocol. Thereafter, an interface at the server pc is programmed in VB and is used to implement the AS/RS system by sending a command to the PLC controller. The abstract of the related interface program built in the server pc is depicted in Fig. 8. To facilitate communication between the PLC and the pc via a RS232/RS422, the picket format for the MISTISUE FX2n-PLC primary port's communication is ruled and shown in Table 2. The definition of the picket format is depicted in Table 3. In addition, the MISTISUE FX2n-PLC extended port's communication is ruled and shown in Table 4 . The communication parameter used in the extended port is shown in Table 5. Finally, the command description of 
the MISTISUE FX2n-PLC extended port's communication protocol type 1 is illustrated in Table 6 .

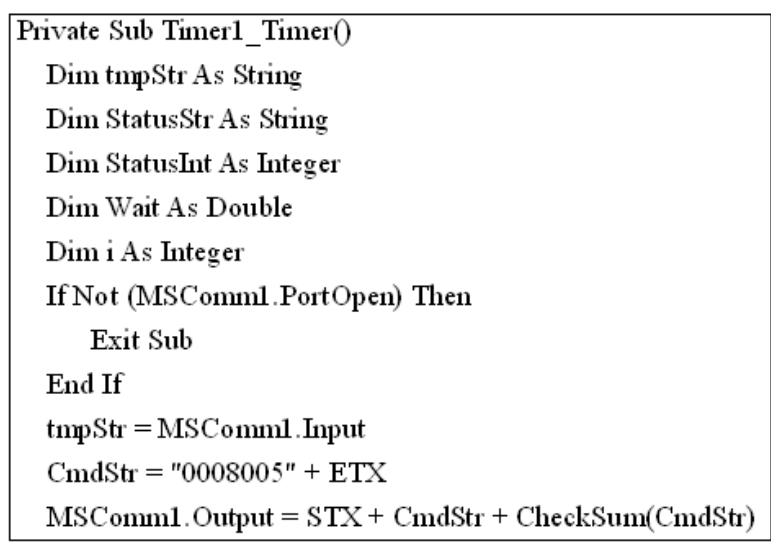

Fig. 8. The abstract of the related interface program built in the server pc.

Table 2. The communication packet format of MISUBISHI FX2n-PLC's primary port.

\begin{tabular}{|c|c|c|c|c|}
\hline $\begin{array}{c}\text { Header } \\
\text { code }\end{array}$ & $\begin{array}{c}\text { Command } \\
\text { code }\end{array}$ & $\begin{array}{c}\text { Address } \\
\text { and data }\end{array}$ & $\begin{array}{c}\text { End } \\
\text { code }\end{array}$ & $\begin{array}{c}\text { Inspection } \\
\text { code }\end{array}$ \\
\hline STX $(02)$ & CMD & DATA & ETX $(03)$ & Checksum \\
\hline
\end{tabular}

Table 3. The definition of communication packet for MISUBISHI FX2n-PLC's primary port.

\begin{tabular}{|c|c|c|c|}
\hline $\begin{array}{c}\text { Command } \\
\text { category }\end{array}$ & $\begin{array}{c}\text { Command } \\
\text { code }\end{array}$ & object & $\begin{array}{c}\text { Function } \\
\text { description }\end{array}$ \\
\hline Read & 0 & $\mathrm{X}, \mathrm{Y}, \mathrm{M}, \mathrm{S}, \mathrm{T}, \mathrm{C}, \mathrm{D}$ & $\begin{array}{c}\text { Read the } \\
\text { object } \\
\text { value }\end{array}$ \\
\hline Write & 1 & $\mathrm{Y}, \mathrm{M}, \mathrm{S}, \mathrm{T}, \mathrm{C}, \mathrm{D}$ & $\begin{array}{c}\text { Write data } \\
\text { to the } \\
\text { object }\end{array}$ \\
\hline Forcing on & 7 & $\mathrm{Y}, \mathrm{M}, \mathrm{S}, \mathrm{T}, \mathrm{C}$ & $\begin{array}{c}\text { Forcing } \\
\text { node to be } \\
\text { on }\end{array}$ \\
\hline Forcing off & 8 & $\mathrm{Y}, \mathrm{M}, \mathrm{S}, \mathrm{T}, \mathrm{C}$ & $\begin{array}{c}\text { Forcing } \\
\text { node to be } \\
\text { off }\end{array}$ \\
\hline
\end{tabular}

Table 4. The communication packet format of MISUBISHI FX2n-PLC's extended port.

\begin{tabular}{|c|c|c|c|c|c|c|}
\hline $\begin{array}{c}\text { Head } \\
\mathrm{er} \\
\text { code }\end{array}$ & $\begin{array}{c}\text { Statio } \\
\mathrm{n} \\
\text { numb } \\
\mathrm{er}\end{array}$ & $\begin{array}{c}\text { Comput } \\
\text { er } \\
\text { number }\end{array}$ & $\begin{array}{c}\text { comma } \\
\text { nd }\end{array}$ & $\begin{array}{c}\text { Object } \\
\text { number/d } \\
\text { ata }\end{array}$ & $\begin{array}{c}\text { En } \\
\mathrm{d} \\
\mathrm{cod} \\
\mathrm{e}\end{array}$ & $\begin{array}{c}\text { Inspecti } \\
\text { on code }\end{array}$ \\
\hline
\end{tabular}

Table 5. The parameter of the extended port's communications protocol for the MISUBISHI FX2n-PLC.

\begin{tabular}{|c|c|c|}
\hline D8120Bit & Name & Content \\
\hline 15 & Protocol type & $\begin{array}{l}0: \text { type } 1 \\
1: \text { type } 4\end{array}$ \\
\hline 14 & protocol & $\begin{array}{l}0: \text { No } \\
1: \text { Yes }\end{array}$ \\
\hline 13 & Inspection code & $\begin{array}{c}0: \text { manipulate } \\
1: \text { auto }\end{array}$ \\
\hline 12 & Type of control & none \\
\hline
\end{tabular}

\begin{tabular}{|c|c|c|}
\hline & line(II) & \\
\hline 11 & $\begin{array}{l}\text { DTR inspection } \\
\text { mode }\end{array}$ & none \\
\hline 10 & $\begin{array}{c}\text { Type of control } \\
\text { line(I) }\end{array}$ & none \\
\hline 9 & End code & none \\
\hline 8 & Header code & \multirow{8}{*}{$\begin{array}{c}\text { none } \\
0011: 300 \\
0222: 4800 \\
0100: 600 \\
1000: 9600 \\
0101: 1200 \\
1001: 19200 \\
0110: 2400\end{array}$} \\
\hline 7 & \multirow{7}{*}{$\begin{array}{c}\text { Transmission } \\
\text { speed }\end{array}$} & \\
\hline 6 & & \\
\hline 5 & & \\
\hline 4 & & \\
\hline & & \\
\hline & & \\
\hline & & \\
\hline 3 & Stop bit & $\begin{array}{l}0: 1 \text { bit } \\
1: 2 \text { bits }\end{array}$ \\
\hline 2 & \multirow[t]{2}{*}{ Parity check } & \multirow{2}{*}{$\begin{array}{c}00: \text { none } 02: \\
\text { even } \\
01: \text { odd }\end{array}$} \\
\hline 1 & & \\
\hline 0 & Data bit & $\begin{array}{l}0: 7 \text { bits } \\
1: 8 \text { bits }\end{array}$ \\
\hline
\end{tabular}

Table 6. The command of the extended port's communications protocol for the MISUBISHI FX2n-PLC.

\begin{tabular}{|c|c|c|}
\hline Command & Description & Available object \\
\hline BR & $\begin{array}{c}\text { Read PLC's bit } \\
\text { data }\end{array}$ & X, Y, M, S \\
\hline WR & $\begin{array}{c}\text { Read PLC's } \\
\text { bit_set data } \\
\text { Write data to } \\
\text { PLC's bit }\end{array}$ & D, TN, CN \\
\hline WW & $\begin{array}{c}\text { Write data to } \\
\text { PLC's bit_set }\end{array}$ & D, TN, CN \\
\hline BT & $\begin{array}{c}\text { Hybrid type of the } \\
\text { writing for bit }\end{array}$ & Y, M, S \\
\hline & $\begin{array}{c}\text { Hybrid type of the } \\
\text { writing for bit_set }\end{array}$ & D, TN, CN \\
\hline & $\begin{array}{c}\text { Actuate PLC's } \\
\text { running }\end{array}$ & \\
\hline & $\begin{array}{c}\text { Actuate PLC's } \\
\text { stop }\end{array}$ & \\
\hline & $\begin{array}{c}\text { Read the PLC's } \\
\text { category }\end{array}$ & \\
\hline & \\
\hline
\end{tabular}

\section{ZigBee wireless network}

ZigBee is a short-distance communication technique. It is based on the Physical Layer (PHY), the Media Access Control Layer (MAC), and the Data Link, which are specified by the IEEE 802.15.4. Moreover, the logic network, data transmission, applied interface specification, and the communication specification between the products are specified by the ZigBee alliance.

The effective distance for the ZigBee transmission is 150 200 meters. The data transmission rate is $20 \mathrm{~K} \sim 250 \mathrm{~K}$ bps. Because it has a little lower effective transmission distance and data transmission rate than the $\mathrm{Wi}-\mathrm{Fi}$ and the Blue-tooth, the ZigBee is confined to the lower data transmission. However, low electricity exhaust, large network nodes, and low device cost are the advantages of ZigBee. ZigBee has been widely adopted in the field of environmental monitoring, 
automatic control, plant monitoring process, building automation, and medical sensoring.

The ZigBee wireless module used in this paper is the IP-Link series (Helicomm brand). It provides a RS232/RS-485 interface. In addition, it adopts a full mesh in the network structure. It also possesses Master/Slave characteristics and two-way communication. The frequency of $900 \mathrm{MHz}$ and $2.4 \mathrm{GH}$ (free of charge) are also used.

IP-Link provides three kinds of modes for the client: the binary protocol mode, the transparent-broadcast mode, and the transparent $-\mathrm{P} 2 \mathrm{P}$ mode.

\subsection{Binary protocol mode}

Based on the command format of the ZigBee, a binary command is written by the user. The data packet submitted from the pc (server pc) will be transmitted to the IP-Link Master via the RS-232/RS-485 protocol. Thereafter, according to the communication format, the IP-Link Master will send the data to the specified IPLink Client. The IP-Link Client will be set to routinely send back the data to the IP-Link Master. The related data will also be forwarded to the pc (server pc) via the RS-232/RS-485 protocol.

\subsection{Transparent-broadcast mode}

Regardless of the ZigBee's command format, the user can freely submit the data to the IP-Link Master via the RS-232/RS-485 protocol. The IP-Link Master will then send the data to all the IP-Link Clients that are linked in the ZigBee network. Similarly, all the IP-Link Clients will be preset to send back the data to the IP-Link Master. The IP-Link Master will forward the data to the $\mathrm{pc}$ (server pc) via the RS-232/RS-485 protocol.

\subsection{Transparent - P2P mode}

Regardless of the ZigBee's command format, the user can freely submit the data to the IP-Link Master via the RS-232/RS-485 protocol. The IP-Link Master will then send the data to the specified IP-Link Clients. The IPLink Client will be preset to send back the data to the IPLink Master. The related data will also be forwarded to the pc (server pc) via the RS-232/RS-485 protocol.

Here, the transparent - P2P mode is adopted in this paper. The ZigBee wireless network can be virtualized as the wireless RS-232/RS-422 tunnel.

\section{Continuous inquiry for the individual AS/RS system}

In order to realize the stock status of all the AS/RS systems and to deploy the appropriate stock between the AS/RS systems, a continuous inquiry from the command control is compulsory. The architecture of the remotely distributing, monitoring, and control network for the multiple AS/RS systems is shown in Fig. 3. All the messages will be sent to the client pc (command control) via TCP/IP network $[10,11,12]$.

\section{The program design for the serial communication between the server port and the client port}

The TCP/IP communication between the server pc and the client pc is performed using the WINSOCK object. The host (server pc) can also be connected simultaneously by the multiple client pcs. The related communication diagram is shown in Fig. 9.

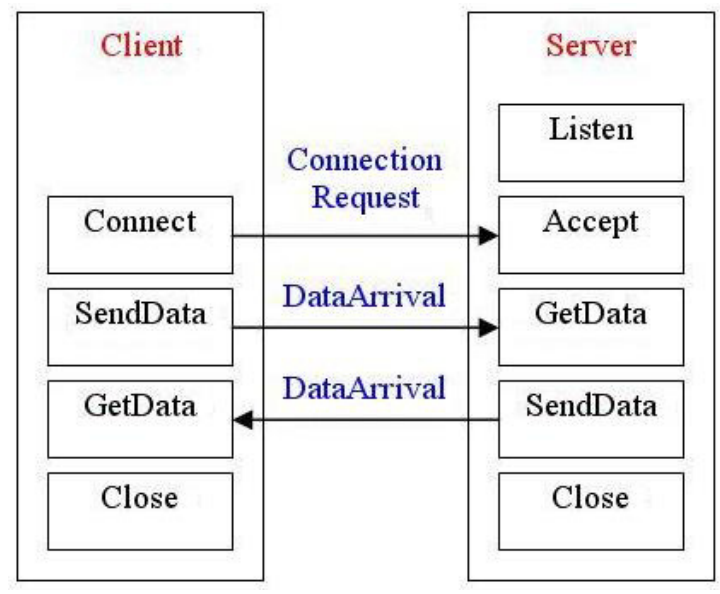

Fig. 9. The communication process between the client $\mathrm{pc}$ and the server pc.

\subsection{Server}

The description of the communication process (server pc viewpoint) is listed below:

\subsubsection{Listen}

Because the server pc will establish a connection platform (function) for the client $\mathrm{pc}$, the condition of "Listen" for the server $p c$ is necessary. The server $p c$ waits for the connection from the client pc when "Listen" is ready. Moreover, because one WINSOCK object in the server port can be used to provide a connection for only one client, multiple WINSOCKs used in the program are required for a network of multiple clients connected to the server port. Therefore, multiple WINSOCKs will be produced using the "Load" command. One of the WINSOCKs (Winsock1(0)) is responsible for the "Listen" function. A "Local Port" will be preset as the port of "Listen."

\subsubsection{Accept}

The server pc will receive an event "Connection Request" from the client pc when the client pc submits a "Connect" for a further internet connection. The server pc also accepts the client pc's connection request using "Accept" via another WINSOCK. 


\subsubsection{Get data}

The server pc receives an event "Data Arrival" from the client $\mathrm{pc}$ when the client $\mathrm{pc}$ sends data to the server pc. At this time, the server pc will also receive data using "Get Data."

\subsubsection{Send data}

The server pc sends data to the client pc using "Send Data."

\subsection{Client}

The description of connection process (client pc viewpoint) is also listed below:

\subsubsection{Connect}

The WINSOCK in the client pc submits a connection request using "Connect." Here, the WINSOCK will assign the server pc's "Port Number" (at "Listen") to be the value of its "Remote Port."

\subsubsection{Send data}

The client pc sends the data set to the server pc using "Send Data" when the internet connection between the server pc and the client pc is established.

\subsubsection{Get data}

The client pc will produce an event "DataArrival" when the server pc sends a data set to the client pc. For the time being, the client pc receives the data using "Get Data."

\subsubsection{Close}

The end connection using "Close" is required when the communication is finished.

\section{Real time visual monitoring}

In order to double check the stock status and immediately handle the emergent event, an IP CAM visual monitoring system is adopted and shown in Fig. 10 .

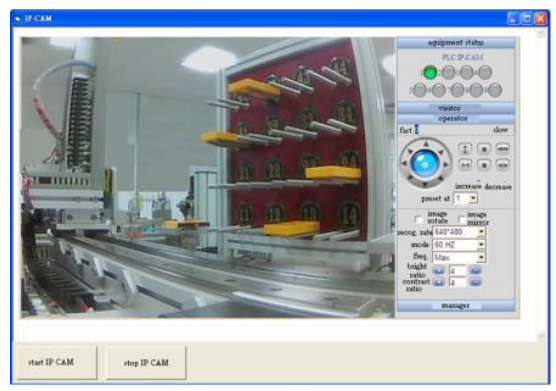

Fig. 10. A IP CAM visual monitoring interface for the AS/RS system.

\section{Results and discussions}

\subsection{Results}

As indicated in Figs. 2 4, a prototype of a remote distributing, monitoring, and controlling network for multiple AS/RS systems with sixteen storage spaces (used for storage/retrieval for one kind of raw material) is established and shown in Fig. 11. A PLC is used as a controller to control the various actuators, including an $\mathrm{X}$-axis, $\mathrm{Y}$-axis, $\mathrm{Z}$-axis air-driven piston, and a grip palm. Two sensors are applied to detect the object at both the feeding and the output platforms. A wireless communication between the server $\mathrm{pc}$ and the AS/RS system is constructed using the ZigBee module connected to the PLC via RS232/RS422 protocol. An interface at the server pc is established to implement the AS/RS system by sending a command to the PLC controller. Moreover, to realize current stock status of all the AS/RS systems and to deploy appropriate stock among the AS/RS systems, a remote distributing, monitoring, and controlling network using a continuous inquiry from the command control via the TCP/IP network is built. As indicated in Fig. 10, to assure the stock's status, a visual monitoring system using the IP CAM is adopted.

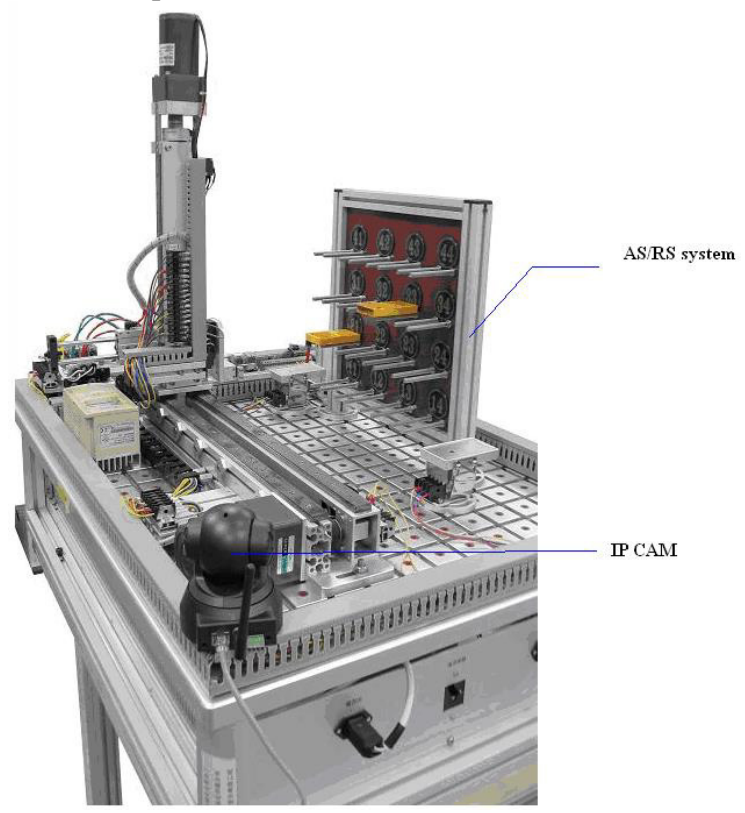

Fig. 11. The prototype of a AS/RS system.

\subsection{Discussions}

The main issues concerning remote wireless monitoring and control storage/retrieval system follow

- Simplifying the search using an AS/RS process with a graph-aided method.

- Establishing the client-server control system between the server pc (local control room for individual AS/RS system) and the client pc (command control). 
- Saving and replacing manpower on site by using an interface between the server pc (local control room) and the AS/RS system.

- Efficient handling and deploying of the appropriate stock between the AS/RS systems using a continuous inquiry at command control (client pc).

- Establishing the hardware of the AS/RS system and accomplishing a communication format between the PLC, ZigBee, and the server pc.

- Assuring stock status and immediately handling an emergent event on site by using a visual monitoring system of IP CAM.

\section{Conclusion}

It has been shown that to reduce cost and to improve the manufacturing process, a remote distributing, monitoring, and controlling network to analyze the online status of multiple AS/RS systems and to remotely deploy stock among the AS/RS systems is necessary. In addition, to avoid interference from internet wiring allocation with existing equipment, a wireless network for the storage/retrieval system using the ZigBee module is adopted. To simplify the search using the AS/RS process with a graph-aided method, a remote distributing, monitoring and controlling network for multiple AS/RS systems with sixteen storage spaces is exemplified and established. The remote distributing, monitoring, and controlling network are utilized using a continuous inquiry between the server pc (local control room for individual AS/RS system) and the client pc (command control) via the TCP/IP network.

Consequently, to assure stock status and quickly respond to emergency events, a visual monitoring system of IP CAM is established.

\section{Acknowledgments}

The authors acknowledge the financial support of the Project (PC101160402).

\section{References}

1. E. Frazelle, Design problems in automated warehousing, Proceeding of IEEE International Conference on Robotics and Automation, 3, 486-489, (1986)

2. T. Suesut, V. Tipsuwanporn, S. Gulphanich, J. Rodcumtui, P. T. Sukprasert, A Design of automatic warehouse for internet based system, Proceedings of IEEE International Conference on Industrial Technology, 313-316, (2002)

3. C. I. Liu, H. Jula, P. A. Ioannou, Design, Simulation, and evaluation of automated container terminals, IEEE Transaction On Intelligent Transportation Systems, 3(1), 12-26, (2002)

4. MURATA MACHINERY, LTD from http://www.muratec-1-system.com/
5. Y. A. Bozer, J. A. White, Design and performance models for end-of-aisle order picking systems, Management Science, 36(7), 852-866, (1990)

6. H. D. Ratliff, A. S. Rosenthal, Order-picking in a rectangular warehouse: a solvable case of the traveling salesman problem, Operations Research, 31, 507-521, (1983)

7. R. Linn, R. A. Wysk, Expert system based controller for an automated storage/retrieval system, I. J. of Production Research, 28(4), 735-756, (1990)

8. P. J. Egbelu, Framework for dynamic positioning of storage/retrieval machines in an automated storage/retrieval system, I. J. of Production Research, 29(1), 17-37, (1991)

9. M. Mansuri, Cycle-time Computation, and dedicated storage assignment, for AS/RS systems, Computer and Industrial Engineering Proceedings of the 1997 1st International Conference on Computer and Industrial Engineering, 33(1/2), 307310, (1997)

10. M. C. Chiu, A multi-function aquarium equipped with automatic thermal control/fodder-feeding/water treatment using network remote controlling system, Information Technology J., 9(7), 1458-1466, (2010)

11. M. C. Chiu, An automatic thermal control on greenhouse using network remote controlling system, J. of Applied Sciences, 10(17), 1944-1950, (2010)

12. M. C. Chiu, T. S. Lan, H. C. Cheng, A three-axis robot manipulation using remote network controlling system, Engineering, 2, 874-878, (2010) 